\title{
加压毛细管电色谱在建立中药黄柏指纹图谱中的 方法学研究
}

吴猗，王彦，谷雪，间超*

上海交通大学药学院, 上海 200240

* 通讯作者, E-mail: chaoyan@unimicrotech.com

收稿日期：2009-05-29；接受日期：2009-06-17

摘要以加压毛细管电色谱( $\mathrm{pCEC}$ ) 为技术平台, 对其在建立中药黄柏指纹图谱中 的方法学进行了研究. 通过对提取溶剂、流动相中有机相种类、盐溶液等条件的优 化, 发现 $1 \%$ 盐酸的甲醇溶液为提取溶液, $20 \mathrm{mmol} / \mathrm{L} \mathrm{NH}_{4} \mathrm{Cl}$ 溶液-乙腈为流动相梯度 洗脱, 紫外检测波长为 $230 \mathrm{~nm}$ 时对其分离效果最好. 并通过在色谱柱上施加不同的 电压, 详细地阐明了 $\mathrm{pCEC}$ 的双重分离机制对分离选择性的影响, 发现黄柏中的主要 成分药根碱、巴马汀和小檗碱在 $\mathrm{pCEC}$ 模式中随电压的不同, 有不同的出峰顺序. 当 电压为 $0 \sim 4 \mathrm{kV}$ 时出峰顺序为药根碱、巴马汀和小檗碱, 当电压为 $8 \sim 14 \mathrm{kV}$ 时出峰顺 序为药根碱、小檗碱和巴马汀. 对此原因进行了详细讨论, 同时与微径液相色谱模式 进行了比较, 说明pCEC可以为复杂样品的分离提供更多更好的分离途径.

\section{1 引言}

毛细管电色谱(Capillary electrochromatography, $\mathrm{CEC})$ 是近年发展起来的一种新型的微分离技术 ${ }^{[1]}$, 是将毛细管柱内填充固定相颗粒、管壁键合固定相或 者制成连续床形式，在电渗流驱动下使样品根据它 们在固定相和流动相中分配系数不同以及电泳速率 不同实现分离. CEC 结合了高效液相色谱 $(\mathrm{High}$ performance liquid chromatography, HPLC)与毛细管 电泳(Capillary electrophoresis, CE)两者的特点, 具有 高柱效、快速分离、高选择性、高精度以及样品用量 少等优点，但单纯的 CEC 模式易产生气泡、干柱等问 题, 而压力驱动的加压毛细管电色谱(pressurized capillary electrochromatography, pCEC)利用电渗流和 压力联合驱动流动相, 能很好地解决上述问题, 且能 实现梯度洗脱 ${ }^{[2]}$, 是电色谱研究领域的一个前沿
技术.

中药由于组成复杂, 高效分离是研究其药效物 质基础至关重要的一步，近年将 CEC这种高效分离 技术用于中药分离逐渐广泛, 而且这些研究也显示 出了 CEC在中药分析中的优越性. 比如刘海兴等使 用开管毛细管电色谱分别测定了旱莲、槐花、槐角中 总黄酮的含量 ${ }^{[3,4]}$, 为药物中黄酮类有效成分的测定 开拓了一种分离效率更高的新方法; 谢国祥等用 pCEC发现西藏红花和普通红花中组分的细微差别, 为全面客观地评价这两种中药材提供了依据 ${ }^{[5]}$.

黄柏是芸香科黄柏属植物黄皮树(川黄柏)或黄 檗(关黄柏)的干燥树皮, 具有清热燥湿、泻火除蒸、 解毒疗疮的功效. 黄柏中主要含小檗碱、木兰碱、黄 柏碱、巴马汀、药根碱等多种生物碱及内酯、甾醇、 黏液质等成分. 众所周知，中药指纹图谱 ${ }^{[6]}$ 是目前国 
际上公认的中药质量评价体系之一，多采用HPLC作 为测定标准. 唐艳梅等也利用 HPLC的方法对黄柏中 的有效成分和指纹图谱进行了研究 ${ }^{[7-9]}$. 建立指纹图 谱的原则是要求其方法具有系统性、特征性和重现性. $\mathrm{pCEC}$ 作为一种新型的高效分离技术, 满足中药指纹 图谱分离的需要，在中药指纹图谱的研究具用很好 的应用前景 $[10,11]$. 在本研究中以 $\mathrm{pCEC}$ 为平台, 针对 黄柏中所含组分的特点, 开展了 $\mathrm{pCEC}$ 在建立黄柏指 纹图谱中的方法学研究. 并比较了微径液相色谱 ( $\mu$-HPLC)和pCEC两种不同分离模式得到的黄柏指纹 图谱的区别, 说明 $\mu-\mathrm{HPLC}$ 和 $\mathrm{pCEC}$ 各自的分离 特点.

\section{2 实验部分}

\section{1 仪器与试剂}

TriSep $^{\mathrm{TM}}$-2100 加压毛细管电色谱仪(UnimicroTechnologies, Inc., Pleasanton, CA, USA)包括 2 台高压 输液洜和溶剂梯度洗脱系统、纳升级定量进样阀、 UV/VIS柱上检测器、双模式高压电源和数据采集分 析系统和 $\mathrm{C} 18$ 毛细管色谱柱(苏州环球色谱公司); KQ2200B型超声波清洗器 (昆山市超声仪器有限公 司); BT 224S电子天平(北京赛多利斯仪器有限公司); $0.22 \mu \mathrm{m}$ 微孔滤膜(醋酸纤维素膜, 上海亚东核级树脂 有限公司); $50 \mu \mathrm{m}$ i.d.熔融石英毛细管柱(河北永年锐 泮色谱器件公司).

乙腈、甲醇为色谱纯(美国 TEDIA 公司); 水为娃 哈哈纯净水; 氯化铵、盐酸为分析纯(国药集团).

黄柏样品购于上海雷允上药店, 盐酸小檗碱 (110713-200609)、盐酸巴马汀(110732200506)、盐酸 药根碱(0733-200005)购自中国药品生物制品检定所.

\section{2 样品溶液制备}

\subsection{1 对照品溶液的配制}

分别称取盐酸小檗碱、盐酸巴马汀、盐酸药根碱 适量, 溶于甲醇中即得, 备用.

\subsection{2 样品供试品溶液的配制}

取黄柏粉末 $0.1 \mathrm{~g}$, 置于 $10 \mathrm{~mL}$ 容量瓶中, 加入 $10 \mathrm{~mL}$ 提取溶剂提取过夜, 再超声 $30 \mathrm{~min}$, 经 $0.22 \mu \mathrm{m}$ 滤膜过滤即得, 备用.

\section{3 色谱条件}

色谱柱: $50 \mathrm{~cm}$ (有效长度 $25 \mathrm{~cm}$ ) $\times 150 \mu \mathrm{m}$ i.d., 5 $\mu \mathrm{m}, \mathrm{C} 18$. 流动相: $\mathrm{A}: 20 \mathrm{mmol} / \mathrm{L} \mathrm{NH}_{4} \mathrm{Cl}$ 溶液-乙腈 (95:5), B: $20 \mathrm{mmol} / \mathrm{L} \mathrm{NH}_{4} \mathrm{Cl}$ 溶液-乙腈(5:95), 洗脱梯 度: $0 \sim 5 \min , 0 \% \sim 5 \% \mathrm{~B} ; 5 \sim 30 \mathrm{~min}, 5 \% \sim 20 \% \mathrm{~B} ; 30$ $\sim 40 \min , 20 \% \sim 35 \% \mathrm{~B}$; $40 \sim 55 \min , 35 \% \sim 50 \% \mathrm{~B}$; $55 \sim 65 \mathrm{~min}, 50 \% \sim 80 \%$ B. 检测波长: $230 \mathrm{~nm}$. 柱上 线性流速: $1.3 \mathrm{~mm} / \mathrm{s}$. 分流比: $500: 1$. 进样量: $1 \mu \mathrm{L}$.

\section{3 结果与讨论}

\section{1 提取溶剂的研究}

黄柏中含有较多生物碱和内酯、甾醇类物质，一 般使用有机溶剂提取能得到较多的提取物. 本实验 中共考察了三种提取溶剂: $95 \%$ 乙腈溶液、甲醇-盐酸 $(99: 1, V / V)$ 溶液和纯甲醇溶液的提取效果, 发现甲 醇-盐酸溶液提取后色谱峰的数量明显多于其他两种 提取溶液提取的效果，故选用其作为提取溶剂.

\section{2 流动相组成的研究}

首先对流动相中有机溶剂的种类和浓度进行了 考察. 实验中所用流动相 $\mathrm{A}$ 为 $20 \mathrm{mmol} / \mathrm{L} \mathrm{NH}_{4} \mathrm{Cl}$-有机 溶剂 $(95: 5, V / V), \mathrm{B}$ 为 $20 \mathrm{mmol} / \mathrm{L} \mathrm{NH} \mathrm{NH}_{4} \mathrm{Cl}$-有机溶剂 $(5: 95, V / V)$. 考察了五种有机溶剂对分离的影响: 甲 醇、甲醇-乙腈 $(75: 25)$ 、甲醇-乙腈 $(50 ： 50$ )、甲醇乙腈 $(25: 75)$ 和乙腈. 发现随着有机溶剂中乙腈比例 的增加, 流动相的洗脱能力加强, 保留时间缩短, 且 基线较平稳, 所以最终选用纯乙腈为流动相的有机 溶剂.

同时对流动相中的盐浓度进行了考察。一般来 说，当流动相中加入一定浓度的 $\mathrm{NH}_{4} \mathrm{Cl}$ 可以提高峰的 分离度, 且使流动相呈酸性, 利于生物碱类物质的分 离. 实验中共选取了 $0,10,20,50,100 \mathrm{mmol} / \mathrm{L}$ 五个 $\mathrm{NH}_{4} \mathrm{Cl}$ 浓度进行比较. 发现当流动相中加入铵盐之后 对分离度及峰的拖尾情况有明显改善，而且盐浓度 越高分离度越好. 但由于在毛细管柱中正常运行的 盐浓度比普通液相色谱低一到两个数量级, 更重要 的是在pCEC加电的情况下, 较高的盐浓度会导致电 流过大而产生比较明显的焦耳热现象和气泡以至于 中断pCEC分析. $20 \mathrm{mmol} / \mathrm{L} \mathrm{NH}_{4} \mathrm{Cl}$ 溶液中对黄柏样品 已经有了较好的分离效果, 更高浓度的盐溶液对其 
分离效果的改善并不十分明显，所以综合考虑最终 使用含 $20 \mathrm{mmol} / \mathrm{L}$ 浓度的 $\mathrm{NH}_{4} \mathrm{Cl}$ 溶液为流动相.

\section{3 检测波长的研究}

黄柏提取物中所含成分复杂, 最大吸收波长差 异很大. 实验中根据所含主要成分的种类，选取了 $210,230,250,270 \mathrm{~nm}$ 四个不同的波长来比较其指纹 图谱. 结果表明波长越小指纹图谱中峰数量越多, 吸 收越好. 但在最小的 $210 \mathrm{~nm}$ 波长时, 基线噪声较大, 溶剂影响也相对比较明显, 所以最终选用 $230 \mathrm{~nm}$ 作 为检测波长。

综合以上条件，在 $230 \mathrm{~nm}$ 波长下，得到黄柏提 取物样品与药根碱、小檗碱和巴马汀三个对照品的 $\mathrm{pCEC}$ 图谱, 如图 1 所示.

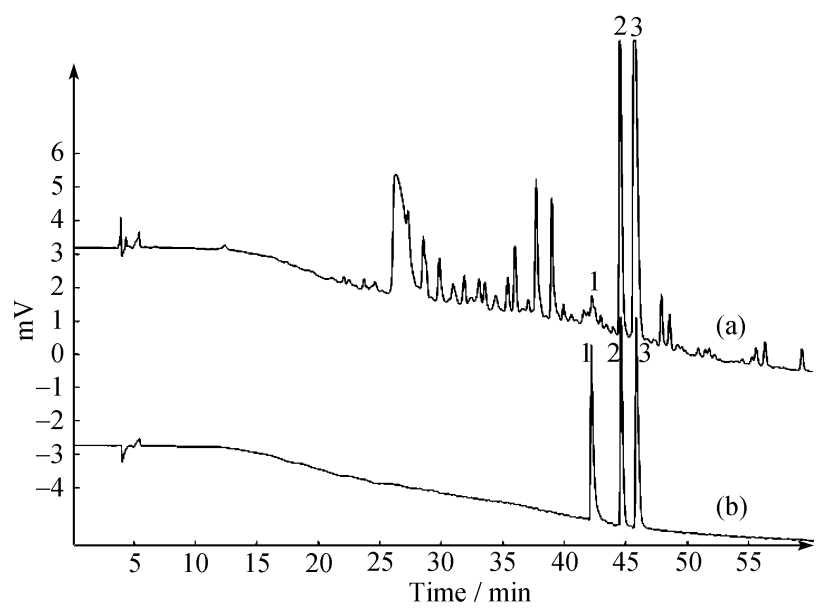

图 1 黄柏样品(a)和对照品(b)的电色谱指纹图

峰: 1. 药根碱 2. 巴马汀 3. 小檗碱.

色谱柱： $55 \mathrm{~cm}$ (有效长度 $30 \mathrm{~cm}$ ) $\times 150 \mu \mathrm{m}$ i.d.毛细管, 填充 $5 \mu \mathrm{m}$ C18; 流动相： $\mathrm{A} ： 20 \mathrm{mmol} / \mathrm{L} \mathrm{NH}_{4} \mathrm{Cl}$ 溶液-乙腈 $(95: 5, \mathrm{~V} / \mathrm{V}), \mathrm{B}: 20$ $\mathrm{mmol} / \mathrm{L} \mathrm{NH}_{4} \mathrm{Cl}$ 溶液-乙腈 $(5: 95, \mathrm{~V} / \mathrm{V})$; 洗脱梯度: $0 \sim 5 \mathrm{~min}, 0 \% \sim$ $5 \% \mathrm{~B} ; 5 \sim 30 \mathrm{~min}, 5 \% \sim 20 \% \mathrm{~B} ; 30 \sim 40 \mathrm{~min}, 20 \% \sim 35 \% \mathrm{~B} ; 40 \sim 55$ $\min , 35 \% \sim 50 \%$ B; $55 \sim 65 \min , 50 \% \sim 80 \%$ B; 流速: $400 \mathrm{~nL} / \mathrm{min}$; 检测波长: $230 \mathrm{~nm}$.

\section{4 施加电压的研究}

\subsection{1 电压对黄柏样品分离的影响}

在加压毛细管电色谱中，电压是控制电渗流大 小和带电物质保留时间的主要因素. 由于生物碱类 物质适合施加正电场，而生物碱类物质是黄柏的主 要成分, 所以本实验装置中柱头连接地电极, 柱尾连
接负电电极.

图 2 是施加不同电压的情况下黄柏样品的 $\mathrm{pCEC}$ 分离图. 图中 1 为盐酸药根碱, 2 为盐酸巴马汀, 3 为 盐酸小檗碱. 从图 2 可以看出, 大多数色谱峰的保留 时间均随电压的增加而变小, 这主要是由于电渗流 随着电压的增加而逐渐增大的缘故.

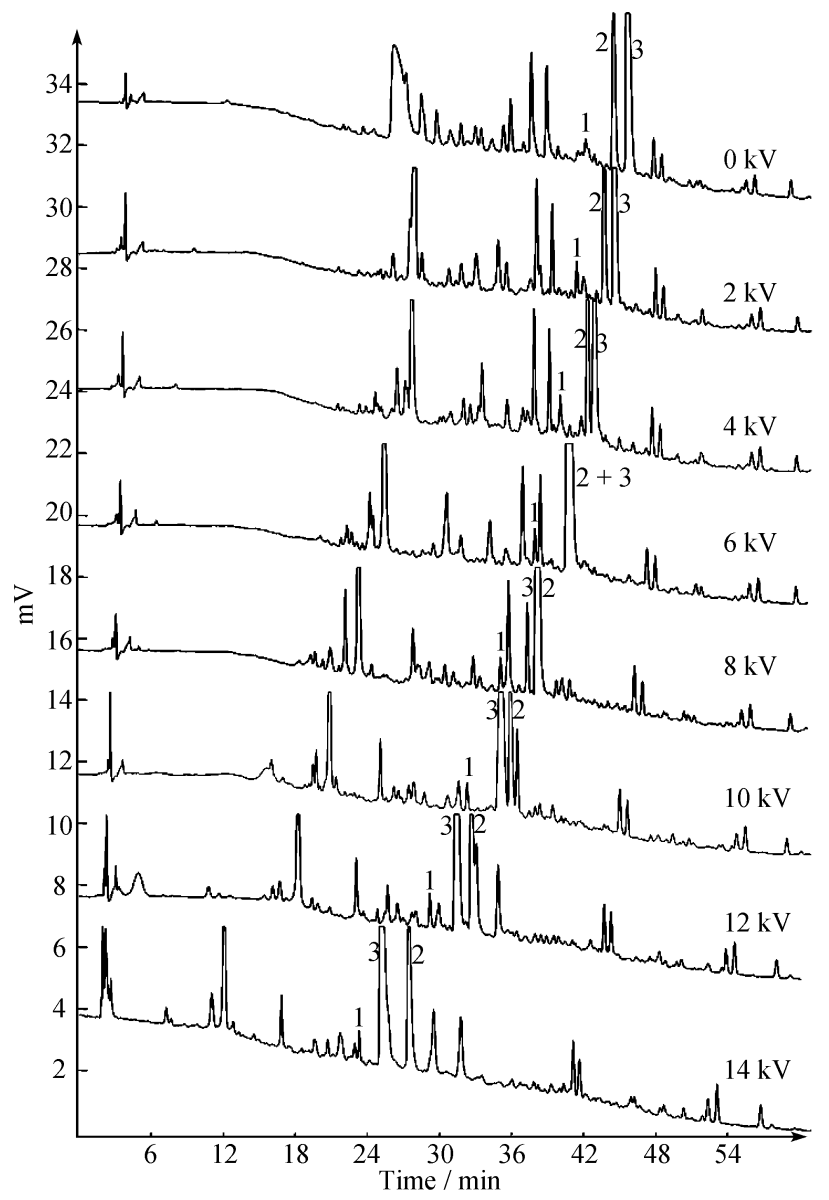

图 2 黄柏样品在不同电压下的电色谱指纹图

峰: 1. 药根碱, 2. 巴马汀, 3. 小檗碱.

其余色谱条件参见图 1

峰容量是表征梯度洗脱时分离度的一个很好的 参数 ${ }^{[12]}$. 本实验通过测定一个梯度时间内平均峰宽 来计算峰容量, 表 1 列出了图 2 中施加不同电压情况 下 $\mathrm{pCEC}$ 分离的峰容量数据. 当电压为 0 时, $\mathrm{pCEC}$ 相 当于微径液相色谱( $\mu$-HPLC)的分离模式, 此时色谱 峰在柱内的流型是抛物线型, 峰较宽, 柱效较低. 所 以在表 1 中, 当电压为 0 时, 峰容量最小. 而加电后, 
表 1 不同电压下 pCEC 峰容量的比较

\begin{tabular}{cc}
\hline 电压 $/ \mathrm{kV}$ & 峰容量 \\
\hline 0 & 66 \\
2 & 72 \\
4 & 77 \\
6 & 77 \\
8 & 79 \\
10 & 81 \\
12 & 102 \\
14 & 96 \\
\hline
\end{tabular}

$\mathrm{pCEC}$ 的分离由压力流和电渗流两方面产生. 电渗流 产生的流型为塞型, 峰较窄, 柱效较高. 所以加电后 以 $\mathrm{pCEC}$ 模式进行分离, 峰容量均高于 $\mu$-HPLC 的分 离模式. 电压为 $12 \mathrm{kV}$ 时, 峰容量最高, 电压为 $14 \mathrm{kV}$ 时，峰容量有所下降，这可以从图 3 的电压-电流曲线 中得到解释. 从图 3 可以看出，溶液的初始电流与电 压成线性增大的关系，说明在此条件下溶液电阻是 符合欧姆定律的. 但当电压继续增大至 $10 \mathrm{kV}$ 以后时, 电流上升逐渐加快, 到 $14 \mathrm{kV}$ 时电流已超过仪器的量 程，说明电压较大时焦耳热效应越来越明显，所以 14 $\mathrm{kV}$ 电压在毛细管柱中产生的焦耳热使得色谱峰有所 展宽，峰容量下降.

同时在图 2 中还可以发现一些有趣的现象，当电 压从 $0 \mathrm{kV}$ 增加到 $4 \mathrm{kV}$ 时, 三种生物碱的出峰顺序由 小至大依次为药根碱、巴马汀和小檗碱; 但当电压增 大至 $8 \mathrm{kV}$ 时, 出峰顺序发生了变化, 从小至大依次 为药根碱、小檗碱和巴马汀. 而且图 2 中巴马汀和小

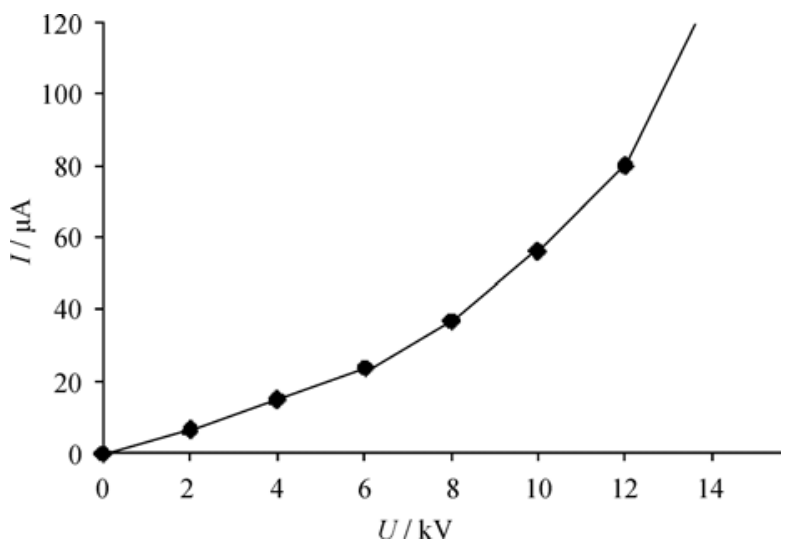

图 3 电流-电压变化曲线
檗碱两个峰的分离度则随电压的增加先减小后增大， 在电压较小时能得到完全分离, 电压增加至 $6 \mathrm{kV}$, 两 个峰重叠在一起, 电压继续增加至 $8 \mathrm{kV}$ 时又能分开, 下面以对照品为例对此现象进行深入的探讨和研究.

\subsection{2 加压毛细管电色谱分离的双重选择性研究}

图 4 是施加不同电压情况下三种生物碱对照品 的 $\mathrm{pCEC}$ 分离图. 图中 1 为药根碱, 2 为巴马汀, 3 为 小檗碱.

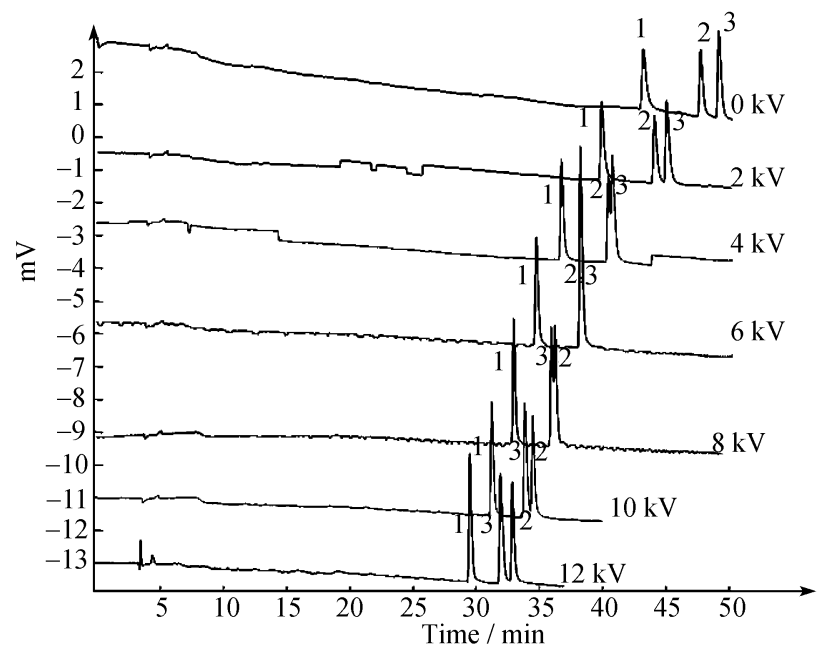

图 4 对照品在不同电压下的电色谱指纹图

峰: 1. 药根碱, 2. 巴马汀, 3. 小檗碱.

其余色谱条件参见图 1

从图 4 可以看出, 三种生物碱的保留时间也均随 电压的增加而变小，而且当电压从 $0 \mathrm{kV}$ 增加到 $6 \mathrm{kV}$ 的范围内, 三种生物碱的出峰顺序由小至大依次为 药根碱、巴马汀和小檗碱; 但当电压增大至 $8 \mathrm{kV}$ 时, 出峰顺序发生了变化, 从小至大依次为药根碱、小檗 碱和巴马汀. 这些现象与图 2 相似.

图 5 为三个对照品药根碱、巴马汀和小檗碱的结 构式. 从结构分析可以看出，这三者的结构比较相似， 很多性质相近, 所以它们的保留时间相差也在 $5 \mathrm{~min}$ 之内. 但结构上的细微差别还是导致这三个对照品 的极性略有不同. 药根碱由于具有酚羟基, 极性最大, 巴马汀次之, 小檗碱最小. 所以在常规的HPLC反相 色谱中, 三个对照品的出峰顺序由其疏水性决定, 为 药根碱、巴马汀、小檗碱 $[13]$. 
<smiles>COc1cc2c(cc1O)CC[n+]1cc3c(OC)c(OC)ccc3cc1-2</smiles>

药根碱

Jatrorrhizine

$\mathrm{C}_{20} \mathrm{H}_{20} \mathrm{NO}_{4}^{+}$<smiles>COc1cc2c(cc1OC)-c1cc3ccc(OC)c(OC)c3c[n+]1CC2</smiles>

巴马汀

Pahmatine

$\mathrm{C}_{21} \mathrm{H}_{22} \mathrm{NO}_{4}^{+}$<smiles>COc1ccc2cc3[n+](cc2c1OC)CCc1cc2c(cc1-3)OCO2</smiles>

小檗碱

Berberine

$\mathrm{C}_{20} \mathrm{H}_{18} \mathrm{NO}_{4}^{+}$

\section{图 5 标准品结构式示意图}

而在普通毛细管电泳中, 物质的保留时间由其 荷质比决定. 这三种生物碱均为季铵碱, 碱性较强, $\mathrm{pKa}$ 在 8 9 左右, 在本实验所用的弱酸性缓冲液环境 下均带正电荷. 另一方面, 由于药根碱的酚羟基在此 环境下虽然不会发生电离, 但会降低药根碱的有效 电荷, 导致其受到的电场力小于小檗碱和巴马汀, 故 其在 $\mathrm{CE}$ 模式中最后出峰. 而巴马汀的分子大于小檗 碱, 即巴马汀在 $\mathrm{CE}$ 中受到的阻力稍大于小檗碱, 所 以这三者在电泳中的出峰顺序为小檗碱、巴马汀、药 根碱 $[14]$.

而 pCEC 结合了普通 HPLC 和毛细管电泳这两者 的特点, 其分离受到 LC 和 CE 双重机制的影响, 当这 两种模式在 $\mathrm{pCEC}$ 中的贡献比例不同时会导致不同 的分离效果. 而调整电压的大小, 就调整了电泳在分 离中做出贡献的比例.

在本实验中当电压为 $0 \mathrm{kV}$ 时, $\mathrm{pCEC}$ 即相当于 $\mu$-HPLC, 所以出峰顺序按疏水性大小依次为药根碱、 巴马汀和小檗碱. 而加入电压以后，电泳在分离中的 贡献逐渐显现. 虽然三个组份的保留时间都随电压 的增加而变小, 但 3 号峰小檗碱的保留时间随电压增 加而变小的程度明显比巴马汀快, 如图 6 所示. 当电 压加至 $4 \mathrm{kV}$ 时, 两者已经不能分开, $6 \mathrm{kV}$ 左右时两者 重叠出峰, 电压再增加至 $8 \mathrm{kV}$ 时, 小檗碱反而在巴 马汀之前出峰. 表 2 比较了在不同电压情况下小檗碱 和巴马汀的分离度, 可以看出, 当电压为 $4,6,8 \mathrm{kV}$ 时, 这两者不能实现分离. 当电压加压 $10 \mathrm{kV}$ 时, 两 者又能基本分开.

可以想象，如果电压能够继续增加的话，当电泳 的作用力成为主导作用时, 三种对照品出峰顺序会 遵从电泳的规律, 依次为小檗碱、巴马汀、药根碱. 但

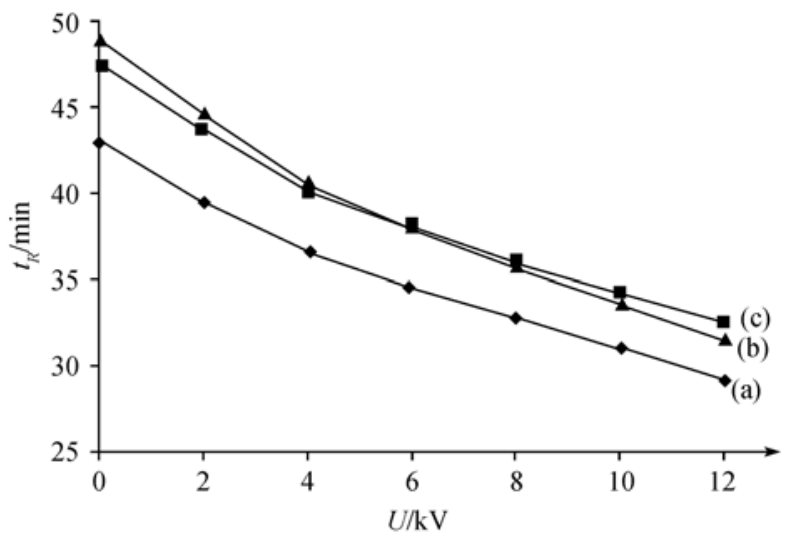

图 6 对照品药根碱(a)、小檗碱(b)和巴马汀(c)保留时间电压变化曲线

表 2 不同电压下巴马汀与小檗碱的分离度的比较

\begin{tabular}{cc}
\hline 电压 $/ \mathrm{kV}$ & $\mathrm{Rs}$ \\
\hline 0 & 2.79 \\
2 & 1.78 \\
4 & 0.94 \\
6 & 0.00 \\
8 & 0.89 \\
10 & 1.59 \\
12 & 2.17 \\
\hline
\end{tabular}

在本次实验中, 由于流动相 $\mathrm{NH}_{4} \mathrm{Cl}$ 导电性较好, 当电 压超过 $15 \mathrm{kV}$ 时, 电流较大, 焦耳热现象明显, 影响 实验进程, 且对色谱柱损伤较大. 所以, 加压毛细管 电色谱的双重保留机制，使它可以在 $\mu$-HPLC的基础 上通过改变电压来调节选择性，此外，通过调节电渗 流与压力的不同比例也能做到微调分离效果.

\section{5 方法学考察}

取供试品溶液连续进样 5 次, 7 个主要峰相对保 
留时间 RSD 小于 $2.1 \%$ ，相对面积 RSD 小于 $4.0 \%$ ，表 明其精密度良好.

取样品 5 份，分别按照供试品溶液的制备方法 制备供试品并测定，7 个主要峰相对保留时间 RSD 小于 $5.0 \%$, 相对面积 RSD 小于 $5.0 \%$, 表明重现性 良好.

取供试品溶液分别在 $0,2,4,8,24 \mathrm{~h}$ 检测, 7 个主 要峰相对保留时间 RSD 小于 $2.3 \%$, 相对面积 RSD 小 于 $4.8 \%$, 表明供试品溶液至少在 $24 \mathrm{~h}$ 内稳定.

\section{4 结论}

$\mathrm{pCEC}$ 作为一种新型的分离技术，具有双重的分
离机理，可以通过电压和压力两方面来调节分离的 选择性. 对黄柏提取液的分离中可以看出, 当不加电 压时，药根碱、小檗碱和巴马汀等物质在 $\mathrm{pCEC}$ 的保 留遵循液相色谱的保留机理; 当施加电压后, 液相的 分配机理与电泳的作用相互竞争，使药根碱、小檗碱 和巴马汀的出峰顺序发生变化, 调节了分离的选择 性. 所以从本文可以看出, $\mathrm{pCEC}$ 能很好地满足建立 指纹图谱所要求的系统性、特征性和重现性的需要, 可以为中药指纹图谱提供一种新的平台技术，尤其 在大批量测定时，其流动相用量少，绿色环保，符合 现代分析技术的发展趋势.

致谢本工作得到 2006 上海市登山行动计划(批准号：061422005)资助，特此致谢.

\section{参考文献}

1 Pretorius V, Hopkins B J, Schieke J D. Electro-osmosis: A new concept for high-speed liquid chromatography. J Chromatogr, 1974, 99: 23-30[DOI]

2 Yao C Y, Gao R Y, Yan C. Quantitative sample injection for capillary electrophoresis. J Sep Sci, 2003, 26(1-2): 37-42[DOI]

3 刘海兴, 刘风芹, 于爱民, 师宇华. 毛细管电色谱法测定旱莲中总黄酮的含量. 滩坊学院学报, 2005, 5(6): 95-96+100

4 刘海兴, 刘风芹, 于爱民, 师宇华, 孙效正, 万丽梅. 毛细管电色谱法测定槐花、槐角中总黄酮含量. 化学试剂, 2006, 28(6): $349-350$

5 Xie G X, Qiu M F, Zhao A H, Jia W. Fingerprint analysis of Flos Carthami by pressurized CEC and LC. Chromatographia, 2006, 64(11-12): 739-743[1DOI]

6 周玉新. 中药指纹图谱研究技术. 北京: 化学工业出版社, 2002

7 丁晴, 徐德然. HPLC 法同时测定黄柏中盐酸药根碱、盐酸巴马汀及盐酸小檗碱的含量. 西北植物学报, 2004，24(11): 2143 - 2145

8 可维, 马春辉, 季宇涁. 不同产地川黄柏 HPLC 指纹图谱的研究. 上海中医药大学学报, 2008, 22(1): 62-65

9 唐艳梅. 黄柏有效成分的含量测定及其指纹图谱研究. 硕士学位论文. 四川：四川农业大学, 2006, 6

10 Xie G X, Zhao A H, Li P, Li L, Jia W. Fingerprint analysis of Rhizoma chuanxiong by pressurized capillary electrochromatography and high-performance liquid chromatography. Biomed Chromatogr, 2007, 21(8): 867-875[DOI]

11 吴孔弦, 谷雪, 阎超. 加压毛细管电色谱法用于银杏叶的指纹图谱研究. 分析化学, 2009, 37(4): 581-585

12 Stoll D R, Paek C, Carr P W. Fast gradient elution reversed-phase high-performance liquid chromatography with diode-array detection as a high-throughput screening method for drugs of abuse I. Chromatographic conditions. J Chromatogr A, 2006, 1137(2): $153-162 \underline{\text { [DOI] }}$

13 杨宏，王天志，常艳波，彭爱华，陈珞. 川黄柏的 HPLC 指纹图谱. 中国天然药物, 2006, 4(5): 360-362

14 胡坤, 赵书林. 黄柏的非水毛细管电泳指纹图谱研究. 分析化学, 2007, 35(6): 857-860 


\title{
Study on pressurized capillary electrochromatography as an fingerprint analytical method for Cortex Phellodendri
}

\author{
WU Yi, WANG Yan, GU Xue \& YAN Chao* \\ School of Pharmacy, Shanghai Jiao Tong University, Shanghai 200240, China
}

\begin{abstract}
Pressurized capillary electrochromatography combines the best features of capillary electrophoresis (CE) and high performance liquid chromatography (HPLC): high separation efficiency, high selectivity, high resolution and fast speed. Besides, it can suppress bubble formation and provides a solvent gradient mode. So pCEC is much more suitable to separate complex system, such as traditional Chinese medicines. The analysis methods of pCEC fingerprint of Cortex Phellodendri, which contain many alkaloid compounds and so on, were studied and optimized. Cortex Phellodendri was extracted by $1 \% \mathrm{HCl}$ methanol solution. $\mathrm{C} 18$ capillary column was used as stationary phase and $20 \mathrm{mmol} / \mathrm{L} \mathrm{NH}_{4} \mathrm{Cl}$ - acetonitrile with gradient as mobile phase. The wavelength of UV detector was $230 \mathrm{~nm}$. In addition, the applied voltage could affect the separation because it was part of the driving force. The value of voltage could control the retention time and the order of some compounds. The order of three standards is jatrorrhizine, palmatine and berberine when the voltage is $0 \sim 4 \mathrm{kV}$, and the order is jatrorrhizine, berberine and palmatine when the voltage is $8 \sim 14 \mathrm{kV}$. This work could provide foundation for more research of fingerprint of Cortex Phellodendri planted in different places.
\end{abstract}

Keywords: pressurized capillary electrochromatography, pCEC, Cortex Phellodendri, fingerprint, selectivity 